\title{
Current Partition: A Nonequilibrium Green's Function Approach
}

\author{
Baigeng Wang, ${ }^{1}$ Jian Wang, ${ }^{1}$ and Hong Guo ${ }^{1,2}$ \\ ${ }^{1}$ Department of Physics, The University of Hong Kong, Pokfulam Road, Hong Kong, China \\ ${ }^{2}$ Center for the Physics of Materials and Department of Physics, McGill University, Montreal, Quebec, Canada H3A 2T8
}

(Received 8 January 1998)

\begin{abstract}
We present a solution to the problem of ac current partition in a multiprobe mesoscopic conductor within the nonequilibrium Green's function formalism. This allows the derivation of dynamic conductance which is appropriate for nonequilibrium situations and which satisfies the current conservation and gauge invariance requirements. This formalism presents a significant generalization to previous theory: (i) There is no limit in the frequency, and (ii) it allows detailed treatments of interactions in the mesoscopic region. The formalism is applied to calculate the dynamic conductance of tunneling structures with and without assuming a wideband limit. [S0031-9007(98)08209-X]

PACS numbers: 73.23.Ad, 72.10.Bg, 73.40.Gk
\end{abstract}

The problem of electric current partition in a mesoscopic multiprobe conductor is a fundamental issue of quantum transport. In the familiar dc situation the issue is well understood from the Landauer-Büttiker formulation [1]. Under time varying ac conditions, this problem becomes more complicated due to the presence of displacement current which is induced by the ac fields according to electrodynamics [2]. In this case, one needs to know how to partition the displacement current in addition to the particle current for each probe of the conductor. Without this knowledge one cannot obtain correct results of dynamic conductance: the electric current will not be conserved unless the displacement current is taken into account. There is another fundamental requirement in the transport which is the gauge invariance of the theory. This simply means that the physics depends only on the voltage difference; thus shifting voltages everywhere by the same constant amount should not not alter the results. In the ac transport the gauge invariance will not be satisfied without taking into account displacement current.

Quantum transport under dynamic conditions is the focus of a number of recent experiments [3-7]. Under low frequency ac fields the system is near equilibrium, the problem of current partition can be analyzed using scattering matrix theory (SMT). Büttiker and co-workers have developed a theoretical formalism $[8,9]$ based on SMT to analyze linear ac transport and derived the dynamic conductance $G_{\alpha \beta}(\omega)$. Here a subscript denotes a probe. By including the contribution of displacement current and its partition among the probes, the derived dynamic conductance guarantees current conservation and gauge invariance [8]. Obviously, displacement current should be even more important for higher frequencies and for situations far from equilibrium. In this Letter we address the problem of current partition far from equilibrium, and with this information we derive a dynamic conductance expression which conserves electric current and satisfies the gauge invariance under nonequilibrium conditions.
Far from equilibrium, the Keldysh nonequilibrium Green's function [10-17] (NEGF) has been widely applied and many problems of great interest have been analyzed. However, within this formalism contribution of displacement current has not been included. In other words the current conservation and gauge invariant condition have not been satisfied [18]. A direct consequence of neglecting displacement current is to predict incomplete results for dynamic conductance, such as that for a parallel plate capacitor. The fact of violating current conservation within the nonequilibrium theories has been recognized in a number of publications $[14,16,19]$. The problem is not solved because of a lack of knowledge for partitioning the displacement current.

Let us consider a quantum coherent multiprobe conductor with the Hamiltonian

$$
\begin{aligned}
H= & \sum_{k \alpha} \epsilon_{k \alpha}(t) c_{k \alpha}^{\dagger} c_{k \alpha}+H_{\mathrm{cen}}\left\{d_{n}, d_{n}^{\dagger}\right\} \\
& +\sum_{k \alpha, n}\left[T_{k \alpha, n} c_{k \alpha}^{\dagger} d_{n}+\text { c.c. }\right],
\end{aligned}
$$

where $\epsilon_{k \alpha}(t)=\epsilon_{k \alpha}^{0}+q V_{\alpha} \cos \omega t$. The first term is the Hamiltonian of probes where the ac signal is applied; the second term is the general Hamiltonian for the scattering region which is a polynomial in $\left\{d_{n}^{\dagger}, d_{n}\right\}$ that commutes with the electron number operator [18] $N=\sum_{n} d_{n}^{\dagger} d_{n}$; the coupling between the probes and the scattering region is given by the last term with coupling matrix $T_{k \alpha, n} . \quad c_{k \alpha}^{\dagger}\left(c_{k \alpha}\right)$ is the creation (annihilation) operator inside the $\alpha$ probe; $d_{n}^{\dagger}\left(d_{n}\right)$ is for the scattering region.

The particle current (conduction current) inside probe $\alpha$ can be calculated using the equation of motion for the number of carriers inside that probe. In terms of the Green's function and the self-energy, it can be written in the familiar form $[12,14]$ in frequency representation $(\hbar=1)$, 


$$
\begin{aligned}
& I_{\alpha}^{c}(\omega)=-q \int_{-\infty}^{+\infty} \frac{d E d E_{1}}{(2 \pi)^{2}} \operatorname{Tr}[ G^{r}\left(E+\omega, E_{1}\right) \Sigma_{\alpha}^{<}\left(E_{1}, E\right)+G^{<}\left(E+\omega, E_{1}\right) \Sigma_{\alpha}^{a}\left(E_{1}, E\right) \\
&\left.-\Sigma_{\alpha}^{<}\left(E+\omega, E_{1}\right) G^{a}\left(E_{1}, E\right)-\Sigma_{\alpha}^{r}\left(E+\omega, E_{1}\right) G^{<}\left(E_{1}, E\right)\right],
\end{aligned}
$$

where the Green's function and self-energy are defined in the usual manner $[12,14]$. The double-time Fourier transform is defined as

$$
F\left(E_{1}, E_{2}\right)=\int d t_{1} \int d t_{2} F\left(t_{1}, t_{2}\right) \exp \left[i\left(E_{1} t_{1}-E_{2} t_{2}\right)\right] .
$$

We will be interested in the current component linear in voltage [20]; hence we simplify Eq. (2) by keeping the first order in voltage. The dynamic conductance coefficient $G_{\alpha \beta}^{c}(\omega)$ due to the particle current is defined as $I_{\alpha}^{c}(\omega)=\sum_{\beta} G_{\alpha \beta}^{c}(\omega) V_{\beta}(\omega)$. $G_{\alpha \beta}^{c}(\omega)$ is just the frequency dependent admittance matrix due to particle current. Without introducing confusion, throughout the following we shall use notation $G$ with subscripts to denote conductance, while that without subscripts (unless otherwise stated) to denote Green's function.

In the presence of ac fields, the particle current alone is not conserved due to charge accumulation in the scattering region $Q(t)$, i.e., $\sum_{\alpha} I_{\alpha}^{c} \neq 0$. It is the total current which is conserved [14], $\sum_{\alpha} I_{\alpha}^{c}(t)+d Q(t) / d t=$ 0 , where $I^{d}=d Q / d t$ is the displacement current. In Fourier space,

$$
\sum_{\alpha} I_{\alpha}^{c}(\omega)=i \omega Q(\omega)
$$

where

$$
Q(\omega)=-\sum_{\beta} i q \int \frac{d E}{2 \pi} \operatorname{Tr}\left[g_{\beta}^{<}(E+\omega, E)\right] V_{\beta}
$$

is the ac charge accumulation in the scattering region and $g_{\beta}^{<}$is the small-signal component of the Green's function $G^{<}$defined as $\sum_{\beta} g_{\beta}^{<} V_{\beta}=G^{<}-G_{e q}^{<}$in the linear regime [14]; here $G_{e q}^{<}$is for equilibrium. This charge is related to the displacement field $\mathbf{D}$ of the internal Coulomb potential via Poisson equation [9] (more generally the potential satisfies Helmhotz equation): $\nabla$. $\mathbf{D}(\mathbf{r}, \boldsymbol{\omega})=4 \pi \rho(\mathbf{r}, \omega)$ with $Q=\int \rho d \mathbf{r}$. Hence the displacement current $-i(\omega / 4 \pi) \int \mathbf{D} d \mathbf{r}$ is just the time derivative of the pileup charge: $\sum_{\alpha} I_{\alpha}^{d}=d Q / d t$. Thus the total current in probe $\alpha$ is $I_{\alpha}^{\text {tot }}=I_{\alpha}^{c}+I_{\alpha}^{d}$. Current conservation means $\sum_{\alpha} I_{\alpha}^{\text {tot }}=0$.

From Eq. (3), only the total displacement current $I^{d}(\omega)=-i \omega Q(\omega)$ is known. We thus need to partition it into each probe $\alpha$. To give an example, the easiest situation would be the wideband limit where the coupling constants $\Gamma_{\alpha}$ are independent of energy. Hence, for an amount of charge $Q$ in the scattering region, the probability of leaking out through probe $\alpha$ is simply $\Gamma_{\alpha} / \Gamma$ with $\Gamma$ the total coupling between the probes to the scattering region. This gives the displacement current partition for each probe: $I_{\alpha}^{d}=\left(\Gamma_{\alpha} / \Gamma\right) I^{d}$.

More generally, we shall use two fundamental requirements of the theory to partition the displacement current: current conservation and gauge invariance. Since current in probe $\alpha$ is $I_{\alpha}=\sum_{\beta} G_{\alpha \beta} V_{\beta}$, current conservation means $\sum_{\alpha} G_{\alpha \beta}=0$, while gauge invariance means $\sum_{\beta} G_{\alpha \beta}=0$. We partition the total displacement current $I^{d}$ into the contributions from individual probes $\alpha$ in the following form: $I_{\alpha}=I_{\alpha}^{c}+A_{\alpha} I^{d}$, or $G_{\alpha \beta}=G_{\alpha \beta}^{c}+$ $A_{\alpha} G_{\beta}^{d}$, where

$$
G_{\beta}^{d}=-q \omega \int \frac{d E}{2 \pi} \operatorname{Tr}\left[g_{\beta}^{<}(E+\omega, E)\right]
$$

is obtained from Eq. (4). The partition coefficients $A_{\alpha}$ must satisfy $\sum_{\alpha} A_{\alpha}=1$ to conserve the total current. To determine $A_{\alpha}$, we apply the gauge invariance condition and obtain $A_{\alpha}=-\left(\sum_{\gamma} G_{\alpha \gamma}^{c}\right) /\left(\sum_{\gamma} G_{\gamma}^{d}\right)$. This gives the following form for the dynamic conductance:

$$
G_{\alpha \beta}=G_{\alpha \beta}^{c}-G_{\beta}^{d} \frac{\sum_{\gamma} G_{\alpha \gamma}^{c}}{\sum_{\gamma} G_{\gamma}^{d}} .
$$

Hence by calculating the Green's functions and using Eqs. (2) and (5), we obtain the dynamic conductance which now satisfies the current conservation and gauge invariance conditions. We further point out that result (6) is quite general: it is suitable for ac transport coefficient in general terms of $\omega$. It can also be applied to the strongly interacting system such as the Anderson model in the large $\mathrm{U}$ limit by including the contribution of displacement current. For that model the Green's functions $G^{<}$and $G^{r}$ have been obtained by $\mathrm{Ng}$ in Ref. [11].

Result (6) is in agreement with the formula derived using SMT, i.e., Eq. (16) of Ref.[8]. However, the quantities involved in Eq. (6) are calculated within NEGF and we now derive these necessary quantities. In the mean field approximation it is straightforward to obtain, from Eqs. (2) and the expression of $I_{\alpha}^{c}(\omega)$,

$$
G_{\alpha \beta}^{c}(\omega)=-q \int \frac{d E}{2 \pi} \operatorname{Tr}\left[\bar{g}_{\beta}^{<}\left(\Sigma_{0 \alpha}^{a}-\bar{\Sigma}_{0 \alpha}^{r}\right)+\bar{g}_{\beta}^{r} \Sigma_{0 \alpha}^{<}-\bar{\Sigma}_{0 \alpha}^{<} \bar{g}_{\beta}^{a}+\left(\bar{G}_{0}^{r} \bar{\sigma}_{\alpha}^{<}-\bar{\sigma}_{\alpha}^{<} G_{0}^{a}+\bar{G}_{0}^{<} \bar{\sigma}_{\alpha}^{a}-\bar{\sigma}_{\alpha}^{r} G_{0}^{<}\right) \delta_{\alpha \beta}\right],
$$

where we have used the abbreviation $g \equiv g(E)$ and $\bar{g} \equiv g(E+\omega)$, etc. In the above equation, $G_{0}$ and $\Sigma^{<, r, a}$ are the equilibrium Green's function and self-energy function; $\bar{\sigma}^{<, r, a}$ is the self-energy function due to the ac field: $\bar{\sigma}_{\alpha}^{<}=(i q / \omega)\left[\Gamma_{\alpha} f-\bar{\Gamma}_{\alpha} \bar{f}\right]$ and $\bar{\sigma}_{\alpha}^{r, a}=(q / \omega)\left[\Sigma_{0 \alpha}^{r, a}-\bar{\Sigma}_{0 \alpha}^{r, a}\right]$, where $f$ is the equilibrium Fermi distribution function (at time $t=-\infty$ ) of the leads far away from the scattering region. We have assumed that there is no dc bias at time $t=-\infty$. The lesser, retarded and advanced Green's functions $\bar{g}^{<, r, a}$ are defined as $\bar{g}_{\alpha}^{r, a}=\bar{G}_{0}^{r, a} \bar{\sigma}_{\alpha}^{r, a} G_{0}^{r, a}$ 
and $\bar{g}_{\alpha}^{<}=\bar{G}_{0}^{r} \bar{\sigma}_{\alpha}^{<} G_{0}^{a}+\bar{G}_{0}^{r} \bar{\sigma}_{\alpha}^{r} G_{0}^{<}+\bar{G}_{0}^{<} \bar{\sigma}_{\alpha}^{a} G_{0}^{a}$. We emphasize that while equilibrium quantities such as the Fermi function do appear in this formalism, they only represent the "initial" condition: the system was in equilibrium at $t=-\infty$. For $t>-\infty$, the nonequilibrium Green's function approach describes the full nonequilibrium dynamic process. With the above definitions and quantities, the dynamic conductance $G_{\alpha \beta}(\omega)$ can be evaluated for a variety of systems near or far from equilibrium.

The wideband limit. - As a first example, it is useful to derive an analytic expression of admittance in the wideband limit. Let us consider the case of resonant tunneling through a quantum dot with a single energy level $E_{0}$. In the wideband limit, the steady state Green's function $G_{0}^{r}$ is

$$
G_{0}^{r}=\frac{1}{E-E_{0}+i \Gamma / 2} .
$$

At zero temperature and through some straightforward algebra we obtain from Eq. (6),

$$
G_{\alpha \beta}=4\left[-\frac{\Gamma_{\alpha} \Gamma_{\beta}}{\Gamma^{2}}+\frac{\Gamma_{\alpha}}{\Gamma} \delta_{\alpha \beta}\right] X,
$$

where $X$ is the ac conductance of a noninteracting (without Coulomb interaction) symmetrical system obtained in Refs. [21,22]. The noninteracting result does not satisfy current conservation and gauge invariance, while the formula (8) does: this is due to the prefactor of $X$ in Eq. (8). One can confirm, at the linear order of ac frequency, that the wideband result (8) agrees with the scattering matrix theory [9] if we assume a Breit-Wigner form of the scattering matrix.

Beyond wideband limit.-As a main result of this work, we now present the dynamic conductance beyond the wideband limit. For this case the situation is quite different and we can obtain results only numerically. From Eq. (6) and the expression of $\bar{\sigma}_{\alpha}^{<}$given above, we also note that due to the energy dependence of $\Gamma$, in general the dynamic conductance depends on the Fermi function in more complicated fashion than simply $d f / d E$. In the following let us calculate the admittance of a symmetric double-barrier resonant tunneling structure which consists of two $\delta$-function barriers with the same strength $V_{o}$. We solve Eq. (6) numerically using a method developed by McLennan et al. [23].

Figure 1 plots $G_{\alpha \beta}(E, \omega)$ as a function of the electron Fermi energy $E$ for three values of $\omega$. Both real and imaginary parts of $G_{21}$ show three peaks due to resonance transmission through the quantum well. For very small $\omega$, e.g., $\omega=0.011$ (solid line), the real part of $G_{21}(E, \omega)$ essentially coincides with the de conductance (not shown); and the imaginary part resembles the emittance obtained from SMT [24]. Substantial deviations are observed from these known limits when $\omega$ is larger. A finite $\omega$ tends to smear out the resonance behavior of the real part of $G_{21}$. In Fig. 2 we plot $G_{21}\left(E_{f}, \omega\right)$ versus $\omega$ for $E_{f} \approx 0.15$ which is near the first resonance energy of Fig. 1. The extra peaks of

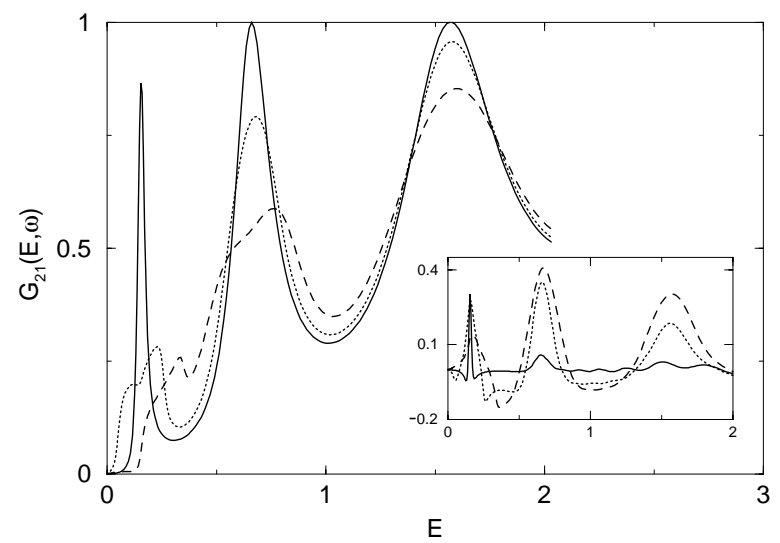

FIG. 1. The real part of $G_{21}(E, \omega)$ as a function of $E$ for three values of $\omega$. Solid line: $\omega=0.01135$; dotted line: $\omega=0.1022$; dashed line: $\omega=0.193$. Inset: the corresponding imaginary part. The barriers' strength $V_{o}=8.81$, and the quantum well width is $100 \AA$. Units of $G_{21}$ are $2 e^{2} / h$, of energy $E$ is eV. We have set $\hbar=1$.

Fig. 2 are due to photon assistant tunneling: the electron can absorb a photon of appropriate energy and exit from the tunnel structure at another resonance level. This picture is confirmed by the inset in Fig. 2 where the energy difference and the frequency difference between resonances are found to be roughly linear functions of each other, with some deviation from perfect linear behavior coming from uncertainties due to the width of the peaks. Such a linear behavior has been observed in the experiment of Ref. [7]. Finally, in Fig. 3 we plot $G_{21}\left(E_{f}, \omega\right)$ versus $\omega$ for double barriers with infinite height: For this case there is no dc current which can flow through and the system becomes a parallel plate capacitor. Because of the displacement field, our formalism predicts a nonzero $G_{21}\left(E_{f}, \omega\right)$. We note that not only the imaginary part of $G_{21}$ is nonzero, even the real part is nonzero. The latter effect gives rise

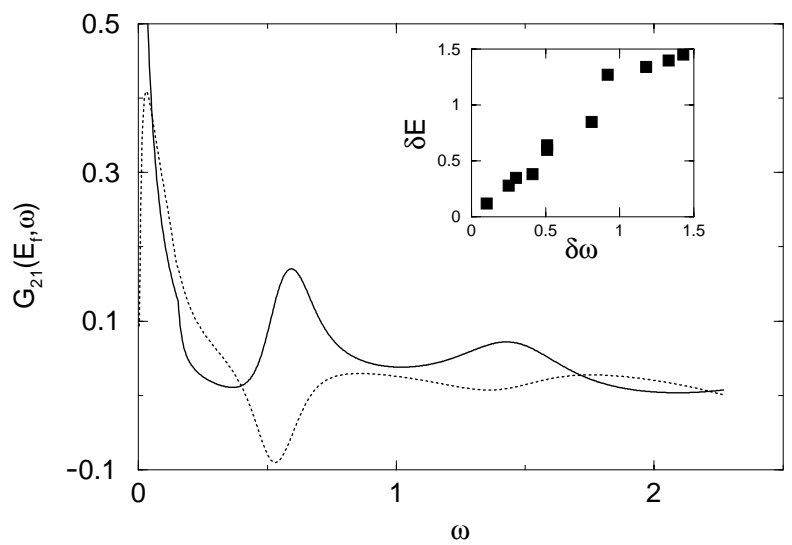

FIG. 2. $G_{21}\left(E_{f}, \omega\right)$ as a function of $\omega$, with $E_{f}=0.1533$. Solid line: read part; dotted line: imaginary part. The peaks are due to photon assisted tunneling. Inset: a roughly linear relationship between the photon energy and the resonance energy. Other system parameters and units are the same as those of Fig. 1. 


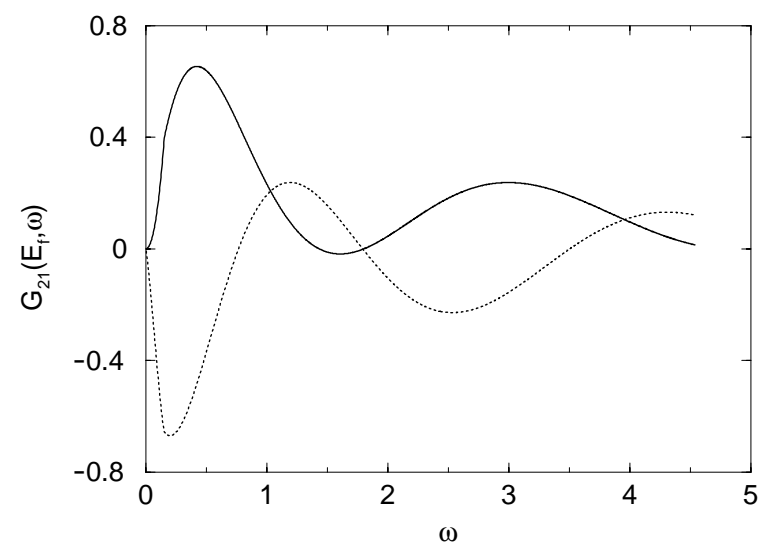

FIG. 3. $G_{21}\left(E_{f}, \omega\right)$ of a parallel plate capacitor as a function of $\omega$ with $E_{F}=0.1533$. The solid (dotted) line is the real (imaginary) part. Other system parameters and units are the same as those of Fig. 1.

to the charge relaxation resistance and is due to contacts implicitly assumed in the transport formalisms [25]. The imaginary part of $G_{21}$ for this system, dividing $\omega$, is just the $\omega$-dependent charge response coefficient. It gives the electrochemical capacitance of the system when $\omega \rightarrow 0$. The oscillatory behavior can be traced back to the retardation effect of Maxwell equations, namely, the Helmhotz equation describing the characteristic potential $[9,26]$ has a term proportional to $\omega^{2}$ which modifies the value and effective sign of the induced charge at large $\omega$, leading to an oscillatory charge response.

In summary, we have provided a solution to the problem of displacement current partition for multiprobe mesoscopic conductors within NEGF. This allows us to obtain dynamic conductance in terms of the ac frequency and the result satisfies the current conservation and gauge invariance conditions. While for special cases (e.g., the wideband limit) analytic results can be obtained, in general the dynamic conductance formula can be evaluated with well established numerical methods. While the nonequilibrium Green's function formalism can be applied to situations far from equilibrium, near equilibrium it must reduce to the same results as obtained from the scattering matrix theory. For the external response, namely, the conductance due to particle current, Ref. [14] has provided this connection. Including the displacement current we have shown, using an explicit example of double barrier tunneling diode, that nonequilibrium Green's function formula indeed recovers the scattering matrix result in the wideband limit where the dynamic conductance becomes a Fermi surface quantity.

We gratefully acknowledgě support by a RGC grant from the SAR Government of Hong Kong under Grant No. HKU 7112/97P, and a CRCG grant from the University of Hong Kong. H. G is supported by NSERC of Canada and FCAR of Québec. We thank the Computing Center of The University of Hong Kong for computational facilities.
Note added.-After the submission of our paper, we became aware of a recent paper [27] which treats photon assisted tunneling in the SMT formulation. In this paper, the internal potential has been considered explicitly and the gauge invariance is satisfied.

[1] R. Landauer, IBM J. Res. Dev. 1, 233 (1957); M. Büttiker, Y. Imry, R. Landauer, and S. Pinhas, Phys. Rev. B. 31, 6207 (1985).

[2] M. Büttiker, Nuovo Cimento Soc. Ital. Fis. 110B, 509 (1995).

[3] J. B. Pieper and J. C. Price, Phys. Rev. Lett. 72, 3586 (1994).

[4] R. A. Webb, S. Washburn, and C.P. Umbach, Phys. Rev. B 37, 8455 (1988).

[5] P. G. N. de Vegvar, G. Timp, P.M. Mankiewich, J.E. Cunningham, R. Behringer, and R. E. Howard, Phys. Rev. B 38, 4326 (1988).

[6] W. Chen, T. P. Smith III, and M. Büttiker, Phys. Rev. Lett. 73, 146 (1994).

[7] T. H. Oosterkamp et al., Phys. Rev. Lett. 78, 1536 (1997).

[8] M. Büttiker, A. Prêtre, and H. Thomas, Phys. Rev. Lett. 70, 4114 (1993).

[9] For a review, see M. Büttiker and T. Christen, in Quantum Transport in Semiconductor Submicron Structures, edited by B. Kramer (Kluwer Academic Publishers, Dordrecht, 1996), p. 263.

[10] N. S. Wingreen, A. P. Jauho, and Y. Meir, Phys. Rev. B 48, 8487 (1993); A. Schiller and S. Hershfield, Phys. Rev. Lett. 77, 1821 (1996).

[11] T. K. Ng, Phys. Rev. Lett. 76, 487 (1996).

[12] A. P. Jauho, N. S. Wingreen, and Y. Meir, Phys. Rev. B 50, 5528 (1994).

[13] H. M. Pastawski, Phys. Rev. B 46, 4053 (1992).

[14] M.P. Anantram and S. Datta, Phys. Rev. B 51, 7632 (1995).

[15] L. Y. Chen and C. S. Ting, Phys. Rev. Lett. 64, 3159 (1990); L. Y. Chen and P.L. Nash, Mod. Phys. Lett. B 11, 35 (1997).

[16] M. H. Hettler and H. Schoeller, Phys. Rev. Lett. 74, 4907 (1995).

[17] G. D. Mahan, Phys. Rep. 145, 251 (1987).

[18] In the nonlinear dc situation, Stafford has considered the gauge invariance in the formalism of NEGF. C.A. Stafford, Phys. Rev. Lett. 77, 2770 (1996).

[19] A. P. Jauho, cond-mat/9711141.

[20] The theory can be generalized to the nonlinear dc case. However even in the linear voltage case, a finite frequency can still drive the system our of equilibrium.

[21] Y. T. Fu and S. C. Dudley, Phys. Rev. Lett. 70, 65 (1993).

[22] C. Jacoboni and P. J. Price, Solid State Commun. 75, 193 (1990).

[23] M. J. McLennan et al., Phys. Rev. B 43, 14333 (1991); also see S. Datta, Electronic Transport in Mesoscopic Systems (Cambridge University Press, New York, 1995).

[24] M. K. Yip, J. Wang, and H. Guo, Z. Phys. B 104, 463 (1997).

[25] M. Büttiker, H. Thomas, and A. Prêtre, Phys. Lett. A 180, 364 (1993).

[26] Z. S. Ma, J. Wang, and H. Guo, cond-mat/9712060.

[27] M. H. Pedersen and M. Büttiker, Phys. Rev. B 58, 12993 (1998). 